\title{
The empirical assessment of multidimensional welfare, inequality and poverty: Sample weighted multivariate generalizations of the Kolmogorov-Smirnov two sample tests for stochastic dominance
}

\section{Gordon Anderson}

Received: 29 November 2004 / Accepted: 8 December 2006 /

Published online: 9 February 2007

(C) Springer Science + Business Media B.V. 2007

\begin{abstract}
Sample weighted multidimensional extensions to existing stochastic dominance, inequality and polarization comparison techniques are introduced and employed to examine whether or not ignoring multidimensional and sample weighting aspects result in misleading inferences. The techniques are employed in the context of a sample of nations, in essence each country in the sample is represented by an agent characterized by the per capita GNP of that country, the GNP growth rate of that country and the average life expectancy in that country. In essence the inequality that is being examined is that between the representative agents in these countries, intra country inequality is not being measured. The results suggest that multidimensional techniques lead to substantially different conclusions from those drawn from the use of unidimensional measures and that sample weighting also has a profound effect on the empirical outcomes.
\end{abstract}

Key words welfare $\cdot$ inequality $\cdot$ poverty

\section{Introduction}

In August 2005 the United Nations Development Program along with others sponsored a conference on "The Many Dimensions of Poverty" highlighting the many circumstances in which the appropriate comparison instruments for evaluating welfare, inequality and poverty are several in number. The general (and diverse) functionings and capabilities approach of Sen [37] and the lifetime wealth approach of Atkinson [7] are two obvious examples. The Atkinson [7] example depends not only on an agent's income stream, but also her life span, the rate at which she discounts happiness through time and the rate at which income may be exchanged through time. Unfortunately, since the appropriate aggregating function of these many instruments (and in the case of poverty comparisons the appropriate function that defines the poverty frontier across the space of instruments) is not directly known or observable, recourse is usually made to unidimensional comparison tests

G. Anderson $(\square)$

Department of Economics, University of Toronto, Toronto, Canada

e-mail: anderson@chass.utoronto.ca 
or indices ${ }^{1}$ of some of the constituent parts individually. Much may be lost in ignoring the multidimensional nature of the issue (by abstracting from the time discounting and life expectancy aspects of lifetime economic wealth for example) and the progress of the welfare, inequality and poverty may be misconstrued as a consequence.

The two basic empirical approaches to making univariate welfare comparisons are through comparing indices and comparing distributions through stochastic dominance tests. Typically indices, such as the Gini coefficient for example, permit a complete ordering of states but can be ambiguous (when Lorenz curves cross it is not clear what differences in the corresponding Gini coefficients represent in welfare terms). Furthermore the statistical distribution of most indices is unknown precluding the possibility of determining whether an ordering is "significant" or just a statistical artifact due to sampling variation. On the other hand dominance tests, based on the examination of dominance orderings, usually provide incomplete orderings but when they are established they are unambiguous and they have well defined statistical properties.

Stochastic Dominance tests are of particular import for poverty analysis since, as Atkinson [9] and Foster and Shorrocks [22] indicate, establishing a dominance relationship between two distributions over a particular range implies an unequivocal change in poverty as measured by all poverty indices within a given class for all potential poverty lines in that range. This is a profoundly liberating result, to a large extent it removes from the arena debates about which poverty line and which poverty index to use and allows attention to be focused upon whether or not poverty has increased or diminished. It transpires that multivariate dominance extensions permit similar assertions for multivariate poverty measures [18]. For poverty analysts this is all the more liberating since now the very tricky problem of defining a poverty frontier (or defining what the various trade-offs between different goods at the poverty boundary would be) can be avoided.

A further issue with both indices and tests is how the sample points are weighted, this has much to do with exactly whose welfare is it that is being represented, but typically little attention has been paid to this issue. As a consequence when welfare across countries is being aggregated or compared the same weight is attached to the per capita GDP of China as that of Ireland in the same way that households with different membership sizes are attached the same weight in un-weighted household studies.

This paper introduces and employs sample weighted multidimensional extensions to existing stochastic dominance comparison techniques to examine whether or not ignoring multidimensional and sample weighting aspects result in misleading inferences. ${ }^{2}$ The techniques are employed in the context of a sample of nations, in essence each country in the sample is represented by an agent characterized by the per capita GNP of that country, the GNP growth rate of that country and the average life expectancy in that country. In essence the inequality that is being examined is that between the representative agents in these countries, intra country inequality is not being measured. Since the welfare of all individuals in the world is at issue each country's representative agent should be weighted by the population she represents. Tests for the comparison of multidimensional distributions in small samples are introduced in Section 2 and Section 3 outlines the theoretical basis for multidimensional comparisons in the application. Section 4 reports the results and some

\footnotetext{
${ }^{1}$ Usually transformations of annual income or consumption measures are used as proxies for lifetime wealth in order to make statements regarding the progress of welfare, inequality and poverty for example.

${ }^{2}$ Multivariate welfare indices already exist (see Tsui [38], Maasoumi [31] and Koshevoy and Mosler [28] for example), however, though straightforward to implement, sample weighted versions of these indices have not generally been employed.
} 
conclusions are drawn in Section 5. The results suggest that multidimensional techniques lead to substantially different conclusions from those drawn from the use of unidimensional measures and that sample weighting also has a profound effect on the empirical outcomes.

\section{Welfare, inequality and poverty tests for multivariate distributions with limited sample sizes}

Since Multidimensional Inequality Indices refer to particular features of the multivariate distribution of characteristics of interest, tests for differences in these distributions would be useful in establishing whether trends in the indices reflect significant changes in the underlying distributions. In the univariate domain, omnibus tests for distributional differences come in two flavours, tests which compare differences between two functions at a sequence of points (like Pearson Goodness of Fit tests) and tests which examine the maximum distance between two functions (like Kolmogorov-Smirnov tests). Rao [36] for example discusses the two tests. Anderson [3] provides a Monte-Carlo based comparison of the two techniques suggesting, amongst other things, superior properties for the latter in detecting location differences and superior properties for the former in detecting scale differences. Generally the choice between the two tests is informed by arguments (a) that the former test is potentially inconsistent whereas the latter is $\operatorname{not}^{3}[11]$ and (b) that the former test is more informative than the latter in highlighting where in the distribution discrepancies occur [36]. More specific unidimensional inequality tests relating to Lorenz, Generalized Lorenz, Stochastic and Polarization Dominance of various orders have been provided in Beach and Davidson [12], Anderson [1, 4, 5], Andrews [6], ([16], Davidson and Duclos [17]), Formby et al. [21], Linton et al. [29], McFadden [30], Barrett and Donald [11].

Crawford [15] and Ibbott [24] extend analogues of the Pearson Goodness of Fit test to the multivariate domain in introducing techniques for making statistical multidimensional stochastic dominance comparisons following the work of Atkinson and Bourguignon [10]. They partition the support of the joint density into many cells and, having estimated the probability of falling in each cell, estimate the relevant cumulative joint and marginal distributions via appropriate aggregation matrices. Unfortunately a curse of dimensionality problem arises since, for precision and power reasons in estimating cell probabilities, it is recommended that partitions should be chosen to ensure a sufficient number of observations (c) are expected to fall into each cell (see Boero et al. [13] for a discussion). Since each dimension requires several cells ${ }^{4}$ (say $r$ ), the demands on the sample size are of the order $c r^{K}$ where $K$ is the number of dimensions.

The Kolmogorov-Smirnov Two Sample Statistic does not suffer this problem since it does not depend upon cell probability calculations. It is readily extended to the multi-variate population weighted framework by using an empirical distribution function $F_{n}\left(\gamma_{x}, X, z\right)$ defined as:

$$
F_{n}\left(\gamma_{x}, X, z\right)=\frac{1}{\sum_{i=1}^{n} \gamma_{i}} \sum_{i=1}^{n} \gamma_{i} I\left(x_{i} \leq z\right)
$$

\footnotetext{
${ }^{3}$ See however Anderson [2].

${ }^{4}$ This issue will always present problems for multidimensional extensions whenever two surfaces are compared using many points of comparison and the number of points of comparison increases with the order of dimensionality such as for example the techniques proposed in Beach and Davidson [12], Davidson and Duclos [16, 17].
} 
where $\gamma_{x}$ is a vector of population weights $\gamma_{i}, X$ is an $n \times K$ matrix of stacked vectors $x_{i}$, $i=1, . ., n$ corresponding to the sample of vectors of $K$ characteristics, $\mathrm{z}$ is a vector of the same dimension as $x_{i}, I()$ is a standard indicator function and " $\leq$ " corresponds to a vector inequality relationship. Defining $G_{m}\left(\gamma_{y}, \mathrm{Y}, \mathrm{z}\right)$ in a similar fashion and letting $n^{*}=n m /(n+m)$, inference can be carried out using $D=\max _{z}\left(F_{n}\left(\gamma_{x}, \mathrm{X}, \mathrm{z}\right)-\mathrm{G}_{\mathrm{m}}\left(\gamma_{\mathrm{y}}, \mathrm{Y}, \mathrm{z}\right)\right)$ or $D_{a}=\max _{\mathrm{z}} \mid F_{n}\left(\gamma_{x}, \mathrm{X}, \mathrm{z}\right)-$ $G_{m}\left(\gamma_{y}, Y, i\right) \mid$. The latter is used to examine unspecified differences in distributions, the former can be used to explore a first order stochastic dominance relationship between $F$ and $G$ by considering $D$ and $\mathrm{DR}=\max _{z}\left(-F_{n}(\mathrm{X}, \mathrm{z})+G_{m}(\mathrm{Y}, \mathrm{z})\right)$. Distribution $G$ first order dominates $F$ when $F\left(x_{1}, x_{2}, . ., x_{K}\right) \geq G\left(x_{1}, x_{2}, . ., x_{\mathrm{K}}\right)$ for all possible $\left(x_{1}, x_{2}, . ., x_{\mathrm{K}}\right)$ with strict inequality holding for some $\left(x_{1}, x_{2}, . ., x_{K}\right)$. Thus $D$ being in the rejection region and DR not being in the rejection region constitutes evidence of first order dominance of $\mathrm{G}$ over $\mathrm{F}$.

For situations where $K=1$ and the $x_{i}$ 's are independent drawings with weights $\gamma_{i}$ from $\mathrm{f}($ ) (and similarly for $\gamma_{j}$ 's) the asymptotic distribution of $D$ and $D_{a}$ in large samples ${ }^{5}$ under a null of identical distributions is given by:

$$
\begin{gathered}
\lim _{n, m \rightarrow \infty} P\left(\sqrt{n^{*}} D<\lambda\right)=1-e^{-2 \lambda^{2}}, \lambda>0 . \\
=0 \text { otherwise } \\
\lim _{n, m \rightarrow \infty} P\left(\sqrt{n^{*}} D_{a}<\lambda\right)=\sum_{k=-\infty}^{\infty}(-1)^{k} e^{-2(k \lambda)^{2}}, \lambda>0 . \\
=0 \text { otherwise }
\end{gathered}
$$

rendering testing non parametric since these distributions are independent of $F$. In the case of $D$ the distribution is particularly simple coming from the family of Rayleigh distributions with scale parameter 0.5 (essentially the underlying random variable would be the positive square root of a $\chi^{2}(2)$ random times 0.5$)$.

Unfortunately in the present circumstances $K>1$ and, since a panel of 135 countries over six observation years is being used, it is extremely unlikely that the sample of $x$ 's will be independent of the sample of $y$ 's. In both cases the distribution of $D$ and $D_{a}$ is not known (recently Linton et al. [29] have attacked the within and between sample dependence issue in $\mathrm{K}-\mathrm{S}$ tests via a sub-sampling approach). Most of the theory on $D$ and $D_{\mathrm{a}}$ has been worked out for the one sample Kolmogorov-Smirnov Test (where $G()$ in the above becomes a theoretical rather than empirical distribution function) and extended to $D$ by invoking Glivenko-Cantelli theorems on the convergence of empirical $G$ to theoretical $G$ asymptotically. Dvoretsky et al. [19] established a probability bound for $D$ in the one sample case when $K=1$ and the realizations are i.i.d, the bound is of the form:

$$
P(\sqrt{n} D>d) \leq \mathrm{Ce}^{-2 d^{2}} \quad n=1,2, . .
$$

Kiefer and Wolfowitz [26] established the existence of a distribution function for the $D \mathrm{~s}$ when $K>1$ but found that generally it depended upon $F$. Kiefer [25] revisited the bounds issue for situations where $K>1$ and established a bound of the form:

$$
P(\sqrt{n} D>d) \leq C(\varepsilon, K) e^{-(2-\varepsilon) d^{2}} \quad \varepsilon>0, n=1,2, . .
$$

Interestingly he observes that $C(\varepsilon, K)<C$ suggesting that estimates of $P(\sqrt{n} D>d)$ for the univariate case would provide conservative (i.e., larger) estimates of the true values when $K>1$. Preliminary simulation studies support this view and suggest that positive correlation

\footnotetext{
${ }^{5}$ The exact distribution of $\mathrm{D}$ is known for $K=1$ and sample sizes smaller than those in the present application (see Kim and Jenrich [27]).
} 
between $x$ and $y$ samples has a similar shrinking effect on the distribution of $D$. Indeed the simulation evidence for positive correlation between the samples is even stronger suggesting that the distribution of $D$ remains in the Rayleigh class and simply reduces the scale parameter. ${ }^{6}$

In studying relative inequality in terms of the multi-variate distributions of characteristics consider the following multi-variate inequality indices. Suppose that sampling is stratified and that a population contains $N$ agent types (note that this is distinct from $n$, the number of agents in the sample) with weights $\gamma_{i}, i=1, . ., N$ where the population weights are such that:

$$
\gamma_{i}>0, i=1 . ., N \text { together with } \sum_{i=1}^{N} \gamma_{i}=N .
$$

A population weighted version of the Tsui multivariate inequality index would take the form:

$$
\left.T R 1 P W=1-\left(\frac{1}{\sum_{i=1}^{N} \gamma_{i}}\right) \sum_{i=1}^{N} \gamma_{i} \prod_{k=1}^{K}\left(\frac{X_{i k}}{\mu_{k}}\right)^{r_{k}}\right)^{\frac{1}{2 r_{k}}}
$$

where the $r_{k}$ are characteristic weights chosen by the investigator and $\mu_{k}$ is the mean of characteristic $k$ (a similar equation for the Maasoumi [33] index may be derived). Alternatively the class of Polarization indices proposed in Esteban and Ray [20] can be extended to many dimensions incorporating a population weighted version of the Koshevoy and Mosler [28] multi-variate Gini coefficient so that POLW, a multivariate version of the Esteban and Ray [20] polarization index with a polarization parameter $\theta$, may be written as:

$$
P O L W=\frac{1}{2 K^{0.5}\left(\sum_{i=1}^{n} \gamma_{i}\right)^{2}} \sum_{i=1}^{N} \sum_{j=1}^{N} \gamma_{i}^{1+\theta} \gamma_{j} \sqrt{\sum_{k=1}^{K}\left(\frac{X_{i k}-X_{j k}}{\mu_{k}}\right)^{2}}
$$

For the pure Koshevoy and Mosler [28] multi-variate Gini coefficient, $\theta$ is set to 0 and $\gamma_{i}=1$ for all $i$. For a polarization index $\theta$ is set to some number between 0 and 1.6 [20] and indicates the degree of polarization reflected in the index $(\theta=0$ corresponds to a standard sample weighted multivariate Gini). Note that if all agents are equally represented, different values of $\theta$ will have no effect and the polarization index reduces to a Gini coefficient.

The important point to observe is that in both of these indices the mean standardized quantities $w_{j k}=x_{j k} / \mu_{k}$ for $j=1, . ., n$ and $k=1, . ., K$ are instrumental and appear in some form. It is their joint distribution that governs the behavior of relative inequality indices and in what follows $f\left(w_{1}, w_{2}, . ., w_{k}\right)$ and $g\left(w_{1}, w_{2}, . ., w_{k}\right)$ will refer to the appropriately transformed distributions. By definition such distributions will not suffer period to period mean location shifts since the expected values of each of their arguments is always the unit vector and a

\footnotetext{
${ }^{6}$ These results are available from the author on request. The intuition behind this is that positively correlated paired elements in the samples would be closer together than if they were independent, reducing the chance of observing large differences in the respective empirical cumulative distribution functions at given points.
} 
strict first order dominance relation will never exist. ${ }^{7}$ Univariate techniques for studying stochastic dominance in these circumstances have been developed [21] but again under extension to multi-dimensional environments they suffer the curse of dimensionality problem alluded to earlier. What is relevant for inequality comparisons like " $f()$ is more equal than $g()$ " is that the terms $\Pi_{k} w_{j k}$ are in some sense closer to 1 under $f()$ than under $g($ ). If there is a significant change in the degree of inequality reflected in the difference between $f()$ and $g(), D_{a}$ can be expected to be significant but it will not indicate the direction of change in inequality. Similarly both $D$ and DR can be expected to be in the rejection region ruling out first order dominance in any direction.

The issue of greater equality under $f()$ than $g()$ is studied by partitioning the sample space and considering the inequalities for the distributions of the mean standardized variates:

$$
\begin{array}{r}
\iint_{00 \ldots 0}^{w_{1}^{*}, w_{2}^{*}, \ldots, w_{K}^{*}} \ldots \int\left(f\left(w_{1}, w_{2}, . ., w_{K}\right)-g\left(w_{1}, w_{2}, . ., w_{K}\right)\right) \mathrm{d} w_{1}, \mathrm{~d} w_{2}, . ., \mathrm{d} w_{K} \leq 0 \\
\text { for all vectors } w * s . t . \prod_{1}^{K} w_{i}^{*} \leq 1 \\
\int_{w_{1}^{*}, w_{2}^{*}, \ldots, w_{K}^{*}}^{\infty \infty, \ldots, \infty} \ldots \int\left(f\left(w_{1}, w_{2}, . ., w_{K}\right)-g\left(w_{1}, w_{2}, . ., w_{K}\right)\right) \mathrm{d} w_{1}, \mathrm{~d} w_{2}, . ., \mathrm{d} w_{K} \geq 0 \\
\text { for all vectors } w^{*} \text { s.t. } \prod_{1}^{K} w_{i}^{*}>1
\end{array}
$$

where strict inequality on the integral conditions holds at least somewhere. Here a partition function has been chosen which exhausts the outcome space and includes the unit vector on the boundary, (all $w^{*}$ such that $\Sigma w^{*}{ }_{i}<K$ could just as well have been chosen as a locus to partition the sample space). The idea is to partition the sample space into two regions, one containing vectors which in some sense are above average across characteristics, the other containing vectors that are below. ${ }^{8}$ Satisfying this condition essentially confirms that the multivariate distribution of $\mathrm{w}$ is more dense around the unit vector under $f(\mathrm{)})$ than under $g(\mathrm{)})$. By considering the transformation $z=-w$, where $f^{-}(z)$ and $g^{-}(z)$ are the appropriate transformations of $f(w)$ and $g(w)$, the second condition may be written as:

$$
\begin{array}{r}
\int_{-\infty}^{z_{1}^{*}, z_{2}^{*} \ldots z_{K}^{*}} \int_{-\infty \ldots-\infty}^{z_{K}} \ldots \int\left(f^{-}\left(z_{1}, z_{2}, . ., z_{K}\right)-g^{-}\left(z_{1}, z_{2}, . ., z_{K}\right)\right) \mathrm{d} z_{1}, \mathrm{~d} z_{2}, \ldots, \mathrm{d} z_{K} \leq 0 \\
\text { for all vectors } z^{*} \text { s.t. } \prod_{i=1}^{\prod_{i}} w_{i}^{*}>1
\end{array}
$$

which, when taken with the first condition, can be seen to be equivalent to two first order stochastic dominance relationships over limited ranges of the relevant distributions which can be examined using the unidirectional versions ( $D$ and DR) of the Kolmogorov-Smirnov test outlined above in each case.

\footnotetext{
${ }^{7}$ This is easily demonstrated for uni-variate distributions $f(w)$ and $g(w)$ confined to the positive orthant since: $E(w \mid f)=E(w \mid g) \rightarrow \int_{0}^{\infty}[(1-F(w))-(1-G(w))] d w=0-\int_{0}^{\infty}(G(w)-F(w)) d w=0$. For the last equality to hold $G(w) \geq F(w)$ for all $0 \leq w$ with strict equality for some $w$ cannot hold and similarly neither can $F(w) \geq G(w)$ for all $0 \leq w$ with strict equality for some $w$.

${ }^{8}$ At first sight it may seem sensible to consider only outcome vectors satisfying $w^{*}{ }_{i} \leq 1$ for $i=1, . ., \mathrm{K}$ (which are on one side of the partition) and outcomes satisfying $w^{*}{ }_{i} \geq 1$ for $i=1, . ., K$ (which are on the other) but to have included only such vectors in the calculus would have ruled out a proportion of the sample space that increases with $K$, (for independent symmetrically distributed characteristics the proportion of observations that satisfy either of the two conditions is on average $(0.5)^{\mathrm{K}-1}$ for example).
} 


\section{An application: Lifetime utility and wealth comparisons}

In unidimensional empirical welfare and inequality comparisons the choice as to which instrument to use depends upon the balance of the arguments for income (that it is more accurately measured) and for consumption (that more adequately reflects an agent's welfare via the consumption smoothing property associated with the permanent income hypothesis see Modigliani and Brumberg [34] and Friedman [23]). The consumption smoothing property emerges from an agent's solution to maximizing lifetime utility constrained by their lifetime income (see Browning and Lusardi [14]). The formulation most favored by researchers assumes a constant relative risk aversion form of an instantaneous utility function (which itself remains unchanged over time) together with the conditions necessary for income smoothing and yields a consumption process of the form:

$$
C_{t}=e^{\frac{\left(g^{*}-r^{*}\right) t}{\zeta}} C_{0}=e^{g t} C_{0} \quad t=0,1, \ldots, T
$$

where the long run consumption growth rate $g^{*}$ (which is equal to the long run interest rate under Phelps' [35] golden rule), the rate of time preference $r^{*}$ and the coefficient of relative risk aversion $\zeta$, imply an incremental consumption augmenting rate of $g=\left(g^{*}-r^{*}\right) / \zeta$.

A more appropriate instrument for welfare, inequality and poverty comparisons is an agent's lifetime wealth or happiness [7]. Given a life length $T$, no bequests and an initial consumption level $C$, lifetime wealth $W$ at $t=0$ may be written as:

$$
W=\int_{0}^{T} C e^{g t} \mathrm{~d} t=\frac{C}{g}\left(e^{g T}-1\right)
$$

In a similar fashion, by employing an instantaneous indirect utility function specification that underlays Working-Leser type Engel curves (utility is linear in the logarithm of consumption) with constant relative prices and time preference discount rates and recalling that $g=\left(g^{*}-r^{*}\right) / \zeta$, an expression for lifetime utility may be obtained as:

$$
U=\int_{0}^{T}\left(a+b \log (C) e^{g^{*} t}\right) e^{-r^{*} t} \mathrm{~d} t=a \frac{\left(e^{-r^{*} T}-1\right)}{-r^{*}}+b \log (c) \frac{\left(e^{\zeta g T}-1\right)}{\zeta g}
$$

Thus with constant long run interest rates and life expectancy across agents, welfare may be reasonably approximated by a linear function of the logarithm of consumption. Since $r^{*}$, $\zeta$ and hence $g$ are fundamentally unobservable neither $W$ or $U$ can be calculated (if they could univariate dominance comparisons could be performed directly). However assuming $r^{*}$ and $\zeta$ are constant and the same for all agents, we can examine the welfare, inequality and poverty implications of changes in $W$ or $U$ by assessing the nature of changes in the joint distribution of their constituent components $g, T$ and $C$. In particular we are interested in the conditions on two joint distributions $f(g, T, C)$ and $g(g, T, C))$ which ensure that either $E_{f}(W)-E_{g}(W)>0$ or $E_{f}(U)-E_{g}(U)>0$.

Extending Atkinson and Bourguignon [10] to the three variable case (where for convenience all of the variables lie in the positive orthant with upper bounds $a_{i}, i=$ $1,2,3$, respectively, and $W_{i}=\partial W / \partial x_{i}, W_{i j}=\partial^{2} W / \partial x_{i} \partial x_{j}$ and $\left.W_{i j k}=\partial^{3} W / \partial x_{i} \partial x_{j} \partial x_{k}\right)$ and letting $\Delta f$ $\left(x_{1}, x_{2}, x_{3}\right)=f\left(x_{1}, x_{2}, x_{3}\right)-g\left(x_{1}, x_{2}, x_{3}\right)$ and $\left.\Delta F\left(x_{1}, x_{2}, x_{3}\right)=F\left(x_{1}, x_{2}, x_{3}\right)-G\left(x_{1}, x_{2}, x_{3}\right)\right)$, 
successive integration by parts yields $\Delta E(W)$ (a similar expression can be derived for $\Delta \mathrm{E}(\mathrm{U}))$ as:

$$
\begin{gathered}
\iint_{0}^{a_{1}} \int_{0}^{a_{2}} \int_{0}^{a_{3}} W\left(x_{1}, x_{2}, x_{3}\right) \Delta f\left(x_{1}, x_{2}, x_{3}\right) \mathrm{d} x_{1} \mathrm{~d} x_{2} \mathrm{~d} x_{3}=W\left(a_{1}, a_{2}, a_{3}\right) \iint_{0}^{a_{1}} \int_{0}^{a_{2}} \int_{0}^{a_{3}} \Delta f\left(x_{1}, x_{2}, x_{3}\right) d x_{1} d x_{2} d x_{3} \\
-\int_{0}^{a_{1}} W_{1}\left(x_{1}, a_{2}, a_{3}\right) \Delta F\left(x_{1}, a_{2}, a_{3}\right) \mathrm{d} x_{1}-\int_{0}^{a_{1}} W_{2}\left(a_{1}, x_{2}, a_{3}\right) \Delta F\left(a_{1}, x_{2}, a_{3}\right) \mathrm{d} x_{2} \\
-\int_{0}^{a_{3}} W_{3}\left(a_{1}, a_{2}, x_{3}\right) \Delta F\left(a_{1}, a_{2}, z_{3}\right) \mathrm{d} x_{3}-\int_{0}^{a_{1}} \int_{0}^{a_{2}} W_{12}\left(x_{1}, x_{2}, a_{3}\right) \Delta F\left(x_{1}, x_{2}, a_{3}\right) \mathrm{d} x_{1} \mathrm{~d} x_{2} \\
+\int_{0}^{a_{1}} \int_{0}^{a_{3}} W_{13}\left(x_{1}, a_{2}, x_{3}\right) \Delta F\left(x_{1}, a_{2}, x_{3}\right) \mathrm{d} x_{1} \mathrm{~d} x_{3}+\int_{0}^{a_{2}} \int_{0}^{a_{3}} W_{23}\left(a_{1}, x_{2}, x_{3}\right) \Delta F\left(a_{1}, x_{2}, x_{3}\right) \mathrm{d} x_{2} \mathrm{~d} x_{3} \\
-\int_{0}^{a_{1}} \int_{0}^{a_{2}} \int_{0}^{a_{3}} W_{123}\left(x_{1}, x_{2}, x_{3}\right) \Delta F\left(x_{1}, x_{2}, x_{3}\right) d x_{1} d x_{2} d x_{3}
\end{gathered}
$$

The first term in this sum is 0 by definition, thus for objective functions in the class $W_{i} \geq$ $0, i=1,2,3, W_{i j} \leq 0 i, j=1,2,3$ and $W_{123} \geq 0$ a sufficient condition for the expected value of the change in objective function to be no lower than 0 is:

$$
\Delta F\left(x_{1}, x_{2}, x_{3}\right) \leq 0 \text { for all } x_{1}, x_{2}, x_{3}
$$

For objective functions in the class $W_{i} \geq 0, i=1,2,3, W_{i j} \geq 0 i, j=1,2,3$ and $W_{123} \geq 0$ (which correspond to the preferences of a risk loving agent in portfolio analysis) a sufficient condition for the expected value of the change in the objective function to be no lower than 0 may be derived by employing the counter cdf $\underline{F}\left(x_{1}, x_{2}, x_{3}\right)=P\left(\left(\mathrm{X}_{1}>x_{1}\right) \cap\left(X_{2}>x_{2}\right) \cap\left(X_{3}>\right.\right.$ $\left.x_{3}\right)$ ). Since the joint density may be written as:

$$
f\left(x_{1}, x_{2}, x_{3}\right)=\frac{\partial^{3} \underline{F}\left(x_{1}, x_{2}, x_{3}\right)}{\partial x_{1} \partial x_{2} \partial x_{3}}
$$

successive integration by parts yields a corresponding expression for $\Delta E(W)$ of the form:

$$
\begin{gathered}
\iint_{0}^{a_{1}} \int_{0}^{a_{2}} \int_{0}^{a_{3}} W\left(x_{1}, x_{2}, x_{3}\right) \Delta f\left(x_{1}, x_{2}, x_{3}\right) \mathrm{d} x_{1} \mathrm{~d} x_{2} \mathrm{~d} x_{3} \\
+\int_{0}^{a_{1}} W_{1}\left(x_{1}, 0,0\right) \Delta \underline{F}\left(x_{1}, 0,0\right) \mathrm{d} x_{1}+\int_{0}^{a_{2}} W_{2}\left(0, x_{2}, 0\right) \Delta \underline{F}\left(0, x_{2}, 0\right) \mathrm{d} x_{2} \\
+\int_{0}^{a_{3}} W_{3}\left(0,0, x_{3}\right) \Delta \underline{F}\left(0,0, x_{3}\right) \mathrm{d} x_{3}+\int_{0}^{a_{1}} \int_{0}^{a_{2}} W_{12}\left(x_{1}, x_{2}, 0\right) \Delta \underline{F}\left(x_{1}, x_{2}, 0\right) \mathrm{d} x_{1} \mathrm{~d} x_{2} \\
+\int_{0}^{a_{1}} \int_{0}^{a_{3}} W_{13}\left(x_{1}, 0, x_{3}\right) \Delta \underline{F}\left(x_{1}, 0, x_{3}\right) \mathrm{d} x_{1} \mathrm{~d} x_{3}+\int_{0}^{a_{2}} \int_{0}^{a_{3}} W_{23}\left(0, x_{2}, x_{3}\right) \Delta \underline{F}\left(0, x_{2}, x_{3}\right) \mathrm{d} x_{2} \mathrm{~d} x_{3} \\
+\int_{0}^{a_{1}} \int_{0}^{a_{2}} \int_{0}^{a_{3}} W_{123}\left(x_{1}, x_{2}, x_{3}\right) \Delta \underline{F}\left(x_{1}, x_{2}, x_{3}\right) \mathrm{d} x_{1} \mathrm{~d} x_{2} \mathrm{~d} x_{3}
\end{gathered}
$$

Noting that all partials of the first three orders are non negative, this will be non-negative if $\underline{F}\left(x_{1}, x_{2}, x_{3}\right) \geq 0$ for all $x_{1}, x_{2}$, and $x_{3}$. Noting that:

$$
\begin{array}{r}
\underline{F}\left(x_{1}, x_{2}, x_{3}\right)=1-F\left(x_{1}, x_{2}, a_{3}\right)-F\left(x_{1}, a_{2}, x_{3}\right)-F\left(a_{1}, x_{2}, x_{3}\right) \\
+F\left(x_{1}, a_{2}, a_{3}\right)+F\left(a_{1}, x_{2}, a_{3}\right)+F\left(a_{1}, a_{2}, x_{3}\right)-F\left(x_{1}, x_{2}, x_{3}\right)
\end{array}
$$


this condition may be written in terms of the cdf's as.

$$
\begin{gathered}
\Delta\left[F\left(x_{1}, x_{2}, x_{3}\right)-F\left(x_{1}, x_{2}, a_{3}\right)-F\left(x_{1}, a_{2}, x_{3}\right)-F\left(a_{1}, x_{2}, x_{3}\right)\right. \\
\left.+F\left(x_{1}, a_{2}, a_{3}\right)+F\left(a_{1}, x_{2}, a_{3}\right)+F\left(a_{1}, a_{2}, x_{3}\right)\right] \\
=K\left(x_{1}, x_{2}, x_{3}\right)-J\left(x_{1}, x_{2}, x_{3}\right) \leq 0 \text { for all } x_{1}, x_{2}, x_{3}
\end{gathered}
$$

where $K()$ is the linear combination of $F$ functions and $J()$ is the corresponding linear combination of $G$ functions. It may be seen that $K\left(x_{1}, x_{2}, x_{3}\right)=1-F\left(x_{1}, x_{2}, x_{3}\right)$ and similarly $J$ $\left.\left(x_{1}, x_{2}, x_{3}\right)=1-\underline{G}\left(x_{1}, x_{2}, x_{3}\right)\right)$. As a consequence $K$ and $J$ take on values in the unit interval and are increasing in each of their arguments ${ }^{9}$ so that the Kolmogorov-Smirnov and other distribution comparison tests would be applicable.

Note the vector of wealth partials with respect to $C, T$ and $g$ in [1] are, respectively:

$$
\begin{gathered}
\frac{1}{g}\left(e^{g T}-1\right) \\
C e^{g T} \\
\frac{C}{g^{2}}\left((g T-1) e^{g T}+1\right)
\end{gathered}
$$

In terms of orders of magnitude, for a wealth augmenting rate of $1 \%$ and an expected lifetime of 60 (roughly the orders of magnitude found in the samples), the partials of $W$ with respect to $C, T$ and $g$ yield elasticities of $1,1.33$ and 0.33 . The matrix of cross partials may be written as:

$$
\begin{aligned}
& 0 \\
& e^{g T} \quad g T e^{g T} \\
& \frac{1}{g^{2}}\left((g T-1) e^{g T}+1\right) \quad T C e^{g T} \quad \frac{C}{g^{3}}\left[(g T)^{2} e^{g T}-2\left((g T-1) e^{g T}+1\right)\right]
\end{aligned}
$$

and:

$$
\frac{\partial^{3} W}{\partial C \partial T \partial g}=T e^{g T}
$$

all of which are positive for pertinent values of the variables. Similar partials and elasticities with respect to $(a+b \ln C), T$ and $g$ as above (with $\zeta g$ replacing $g$ ) can be elicited from (1a) indicating that condition ( $2 \mathrm{a})$ is the one to be examined in the present context.

\section{Results}

Data from the World Bank World Development Indicator series on per capita purchasing power parity GDP in constant 1995 \$US together with Population Size and Life Expectancy were collected for 135 countries for the years 1987, 1990, 1992, 1995, 1997 and 1999 (Appendix contains a list of countries in the sample). GDP per capita growth rates were calculated as the annual average over years since the preceding observation. The

\footnotetext{
${ }^{9}$ This argument was kindly pointed out to me by a referee.
} 
inequality indices require all comparator variables to be non-negative, hence growth rates were standardized in each observation year by deducting the lowest average growth rate in that year from all others so that the variable may be interpreted as the growth rate differential over the minimum growth rate for that observation year. Sample weighting was based upon relative population size each year. Table 1 presents summary statistics for the raw data (i.e. growth rates have not yet been standardized).

Broadly speaking mean lnGDP and median life expectancy grew throughout the period in both weighted and un-weighted terms (there was a slight diminution of un-weighted lnGDP in 1992 and of un-weighted life expectancy in 1999). The trend in median lnGDP was somewhat less categorical, it also fell in 1999. Average and median growth rates increased and then diminished over the period. The effect of sample weighting is evident in diminished lnGDP and life expectancy and increased growth rate means, reflecting the increased weight of less developed countries in the sample. The sample standard deviations, being a crude inequality measure, provide an interesting perspective of increasing inequality in un-weighted lnGNP and diminishing inequality in Weighted lnGNP. Growth rate inequality is all over the map! With respect to life expectancy, inequality increases in the un-weighted sample whilst the weighted sample exhibits a $U$ shaped profile.

Table 2 reports the absolute welfare and poverty implications of the data in Table 1 via multi-variate first order stochastic dominance tests employing criteria (2a). For comparison uni-variate stochastic dominance tests of the first and second orders are reported (both weighted and unweighted). In the multi-variate comparison both un-weighted and weighted samples indicate welfare improvements (poverty reductions) between 1990 and 1992 and 1997 and 1999 and welfare deterioration (poverty increases) between 1992 and 1995 and 1995 and 1997 are indicated by the un-weighted sample but not in the weighted sample (Thus sample weighting can be seen to profoundly affect the results in these comparison years). This suggests that the multidimensional comparison is greatly influenced by the growth rate variable which has the largest coefficient of variation by a considerable margin

Table 1 Sample statistics

\begin{tabular}{|c|c|c|c|c|c|c|}
\hline Year & LnGDP & $\begin{array}{l}\operatorname{lnGDP} \\
\text { (sample weighted) }\end{array}$ & GDPgrowth & $\begin{array}{l}\text { GDPgrowth } \\
\text { (sample weighted) }\end{array}$ & Life expect & $\begin{array}{l}\text { Life expect } \\
\text { (sample weighted) }\end{array}$ \\
\hline \multicolumn{7}{|l|}{ Means } \\
\hline 1990 & 8.3389 & 8.0357 & 0.0014 & 0.0166 & 63.9751 & 65.4408 \\
\hline 1992 & 8.3316 & 8.0851 & -0.0037 & 0.0291 & 64.3698 & 65.9142 \\
\hline 1995 & 8.3499 & 8.1996 & 0.0061 & 0.0416 & 64.6455 & 66.3759 \\
\hline 1997 & 8.3713 & 8.2411 & 0.0107 & 0.0249 & 64.9619 & 66.7446 \\
\hline 1999 & 8.3777 & 8.2681 & 0.0032 & 0.0175 & 64.7490 & 66.7853 \\
\hline \multicolumn{7}{|c|}{ Medians } \\
\hline 1990 & 8.3856 & 7.3709 & 0.0028 & 0.0169 & 68.0839 & 61.7073 \\
\hline 1992 & 8.3623 & 7.5668 & 0.0060 & 0.0101 & 68.1024 & 62.7073 \\
\hline 1995 & 8.3938 & 7.8941 & 0.0116 & 0.0215 & 68.4610 & 64.1459 \\
\hline 1997 & 8.4193 & 8.0193 & 0.0091 & 0.0149 & 69.0366 & 65.1049 \\
\hline 1999 & 8.4118 & 7.8934 & 0.0041 & 0.0116 & 69.4776 & 65.7244 \\
\hline \multicolumn{7}{|c|}{ Sample standard deviations } \\
\hline 1990 & 1.0770 & 1.0491 & 0.0372 & 0.0280 & 10.8973 & 7.8862 \\
\hline 1992 & 1.0823 & 1.0194 & 0.0586 & 0.0518 & 11.1240 & 7.7998 \\
\hline 1995 & 1.1072 & 0.9762 & 0.0447 & 0.0505 & 11.3641 & 7.8112 \\
\hline 1997 & 1.1153 & 0.9690 & 0.0473 & 0.0283 & 11.6624 & 8.0017 \\
\hline 1999 & 1.1299 & 0.9577 & 0.0460 & 0.0373 & 12.4382 & 8.5735 \\
\hline
\end{tabular}




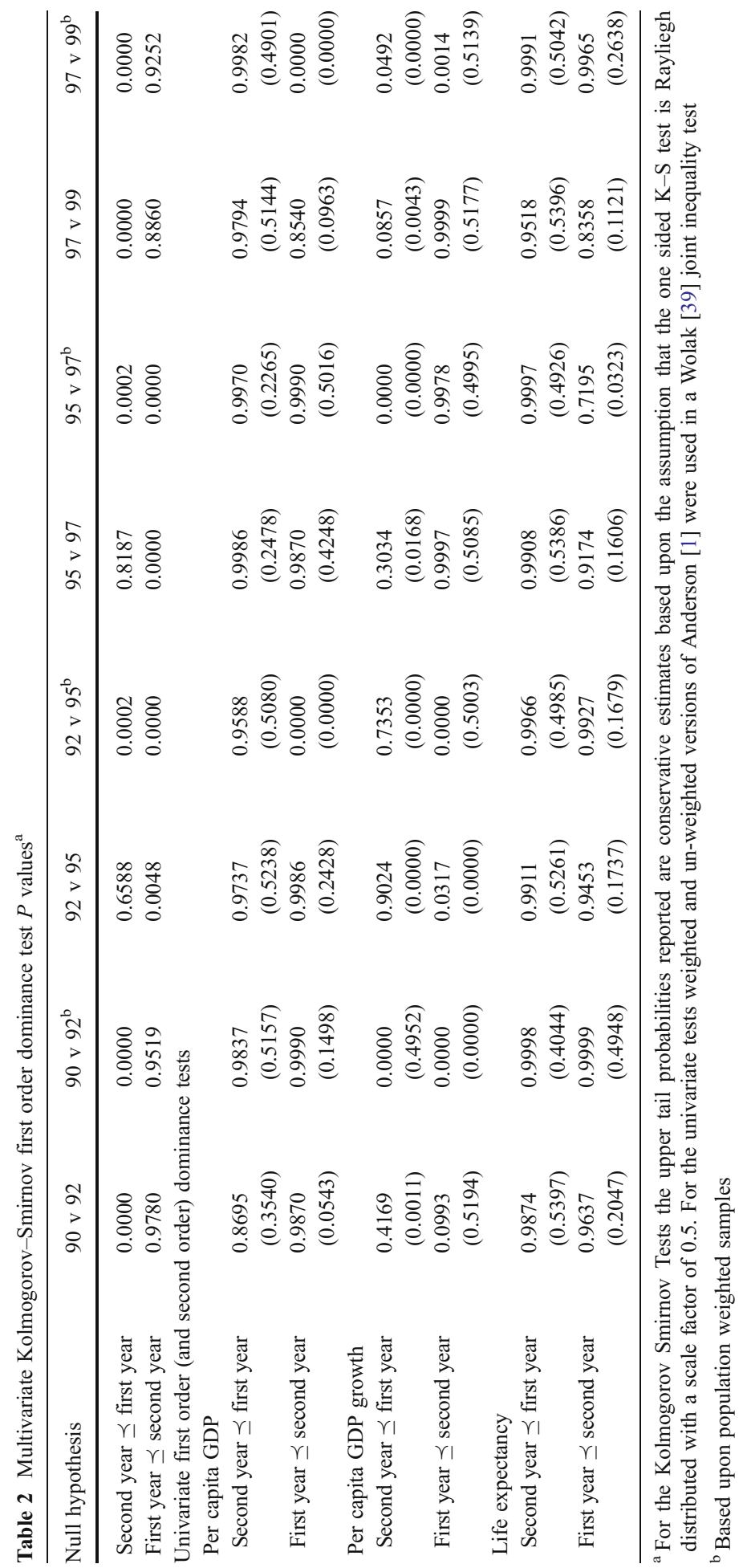


Table 3 Multivariate stochastic dominance test $P$ values for the joint distributions of the mean standardized variables (population weighted results) ${ }^{\mathrm{a}}$

\begin{tabular}{llllll}
\hline Comparison & & $1990 \mathrm{v} 1992$ & $1992 \mathrm{v} 1995$ & $1995 \mathrm{v} 1997$ & $1997 \mathrm{v} 1999$ \\
\hline Overall distrib. & Second $\preceq$ first & 0.0099 & 0.0000 & 0.0001 & 0.0001 \\
& First $\preceq$ second & 0.0973 & 0.9463 & 0.0877 & 0.0846 \\
Lower tail & Second $\preceq$ first & 0.8921 & 0.3028 & 0.9704 & 0.3997 \\
& First $\preceq$ second & 0.2912 & 0.9818 & 0.3550 & 0.6891 \\
Upper tail & Second $\preceq$ first & 0.2685 & 0.6401 & 0.9731 & 0.1006 \\
& First $\preceq$ second & 0.0964 & 0.0020 & 0.0841 & 0.1115 \\
\hline
\end{tabular}
${ }^{\text {a }}$ The upper tail probabilities reported are conservative estimates based upon the assumption that the one
sided K-S test is Rayliegh distributed with a scale factor of 0.5

since it follows the progress of that instrument. Indeed the uni-variate comparisons suggest that it is the growth rate variable that is driving the results since dominance is seldom indicated in the GNP variate (twice) and never in the life expectancy variate. The multivariate results are probably also reinforced by the weak performance of lnGDP in 1992 and both $\operatorname{lnGDP}$ and life expectancy in 1999 as observed in Table 1.

Attention is now turned to the comparison of the joint distributions of the mean standardized variables outlined at the end of Section 1 as a means of examining multivariate inequality changes.

There appears to be stronger evidence of inequality trends in the dominance tests reported in Table 3. Contrary to expectations, at the 5\% level, the general first order dominance tests reject dominance in only one direction whereas the upper and lower tail tests (with the exception of the $92-95$ comparison) have to be deemed inconclusive. ${ }^{10}$ However at the $10 \%$ level general overall distribution first order dominance is rejected in both directions for all but the 1992-1995 comparison and in this context upper tail dominance of the first period over the second period is not rejected whereas the second period's dominance over the first is indicating decreasing inequality over the period which is consistent with the general trend in the sample statistics.

For comparison Table 4 reports results for the various Multidimensional Inequality Indices and their unidimensional counterparts (for the purposes of these results the growth rates have been standardized). The unidimensional inequality indices for lnGDP and Life Expectancy generally follow the patterns indicated by their corresponding sample standard deviation counterparts whereas the inequality indices for growth rates do not, but recall that these have been standardized in each period for the inequality calculations whereas the standard deviations are based upon raw data. Sample weighting again has a profound effect upon the conclusions drawn, effectively reversing the trends observed in lnGDP and Life Expectancy. Since in welfare and inequality calculations sample weighting by population size is appropriate, we now focus upon these results. It is interesting to note that reliance upon any single aspect of the lifetime wealth calculation would lead to quite different inferences from those derived from any other. Population Weighted Gini and Polarization coefficients for lnGDP would suggest a continued and sustained decline of both inequality and polarization whereas inequality and polarization time profiles are $U$ shaped for life expectancy and inverted $U$ shaped for growth. What conclusions are to be drawn when the various trends are taken together? With regard to the multivariate indices it should at first be

${ }^{10}$ The lack of power in the "tail" directed tests is probably due to the reduction in the sample size (it is roughly halved) implicit in focusing on the tails. 
Table 4 Multidimensional inequality indices

\begin{tabular}{|c|c|c|c|c|c|c|c|c|c|}
\hline Year & $\begin{array}{l}\text { Gini } \\
\text { MPOL } \\
\theta=0, \gamma_{i}=1\end{array}$ & $\begin{array}{l}\text { Weighted } \\
\text { Gini } \\
\text { MPOL } \\
\theta=0\end{array}$ & $\begin{array}{l}\text { Polarization } \\
\text { index } \\
\text { MPOL } \\
\theta=1\end{array}$ & $\begin{array}{l}\text { TR1PW } \\
\gamma_{i}=1\end{array}$ & $\begin{array}{l}\text { Weighted } \\
\text { Tsui index } \\
\text { TR1PW }\end{array}$ & & & & \\
\hline 1990 & 0.1270 & 0.0938 & 1.0679 & 0.0157 & 0.0142 & & & & \\
\hline 1992 & 0.1107 & 0.0921 & 1.2154 & 0.0138 & 0.0121 & & & & \\
\hline 1995 & 0.1062 & 0.0999 & 1.3251 & 0.0122 & 0.0106 & & & & \\
\hline 1997 & 0.1315 & 0.0968 & 1.2578 & 0.0167 & 0.0150 & & & & \\
\hline 1999 & 0.1178 & 0.0918 & 1.0886 & 0.0127 & 0.0109 & & & & \\
\hline \multicolumn{10}{|c|}{ Unidimensional Gini (un-weighted and weighted) and Esteban-Ray Indices } \\
\hline \multirow[t]{2}{*}{ Year } & \multicolumn{3}{|c|}{ Ln (per cap GDP) } & \multicolumn{3}{|c|}{ GDP growth } & \multicolumn{3}{|c|}{ Life expectancy } \\
\hline & $\begin{array}{l}\text { MPOL } \\
\theta=0, \gamma_{i}=1\end{array}$ & $\begin{array}{l}\text { MPOL } \\
\theta=0\end{array}$ & $\begin{array}{l}\text { MPOL } \\
\theta=1\end{array}$ & $\begin{array}{l}\text { MPOL } \\
\theta=0, \gamma_{i}=1\end{array}$ & $\begin{array}{l}\text { MPOL } \\
\theta=0\end{array}$ & $\begin{array}{l}\text { MPOL } \\
\theta=1\end{array}$ & $\begin{array}{l}\text { MPOL } \\
\theta=0, \gamma_{i}=1\end{array}$ & $\begin{array}{l}\text { MPOL } \\
\theta=0\end{array}$ & $\begin{array}{l}\text { MPOL } \\
\theta=1\end{array}$ \\
\hline 1990 & 0.0741 & 0.0658 & 0.7301 & 0.1539 & 0.1064 & 1.1673 & 0.0949 & 0.0672 & 0.7929 \\
\hline 1992 & 0.0744 & 0.0648 & 0.7190 & 0.1163 & 0.1070 & 1.5838 & 0.0955 & 0.0651 & 0.7481 \\
\hline 1995 & 0.0759 & 0.0627 & 0.6903 & 0.1030 & 0.1261 & 1.8832 & 0.0974 & 0.0641 & 0.7170 \\
\hline 1997 & 0.0763 & 0.0622 & 0.6828 & 0.1546 & 0.1199 & 1.7484 & 0.0991 & 0.0646 & 0.7068 \\
\hline 1999 & 0.0771 & 0.0615 & 0.6699 & 0.1205 & 0.1068 & 1.3823 & 0.1053 & 0.0685 & 0.7467 \\
\hline
\end{tabular}

observed that the Multivariate Gini and Tsui/Maasoumi indices are in broad agreement, 1995 population weighted and population and characteristic weighted indices being the exception. Generally the Tsui/Maasoumi indices exhibit more variability than the corresponding Mgini index. Given the diverse trends of the components it is no surprise that no solid underlying trend is discernable in the multi-variate indices except for the polarization indices which both exhibit an inverted $U$ time profile.

\section{Conclusions}

Sample weighted tests facilitating the examination of welfare, poverty and inequality in many dimensions have been introduced which, in an example of an international representative agent lifetime welfare model, indicate that substantially different conclusions may be inferred from those drawn from univariate comparisons. The index techniques introduced are simple generalizations of the familiar univariate Gini coefficient which also extend the Esteban and Ray [20] class of polarization indices. These were found to follow closely the behavior of ethically based and generalized entropy multidimensional inequality indices. The testing techniques are multivariate extensions of the Kolmogorov-Smirnov two sample test (Rao [36]) together with a simple adaptation to examine the "multidimensional tail behavior" of mean standardized data employed in inequality studies.

In an application studying income, growth and life expectancy, three variables that influence lifetime wealth, the variables exhibit quite distinct and different inequality and polarization trends over the observation period. It is then perhaps no surprise that the multivariate indices which in essence report an agglomeration of the three indices indicate no such discernable trend except for the $U$ shaped time profile of the polarization indices over the period of study. It is equally unsurprising that the unequivocal welfare improvements inferred from a univariate analysis (see for example Anderson [4]) become much less clear in the multivariate paradigm. Variations in the sample weighting scheme also had a profound impact on the conclusions to be drawn from both indices and tests. 
Acknowledgements Many thanks are due to two anonymous referees, to Charles Beach, Essie Maasoumi, Lars Osberg and Russell Davidson for advice in the early stages of this work, to conference participants at the IARIW General Conference in Cork Ireland, Jean-Yves Duclos, Markus Jantti, Maria Ana Lugo, Jaques Silber, Shlomo Yitzhaki and to Ying Ge for his helpful research assistance. The work has been carried out with the support of the SSHRC under grant numbers 4100000732 and 410040254 . All errors are the responsibility of the author.

\section{Appendix}

Countries represented in the sample

Algeria, Angola, Antigua and Barbuda, Argentina, Australia, Austria, Azerbaijan, Bangladesh, Barbados, Belarus, Belgium, Belize, Benin, Bolivia, Botswana, Brazil, Bulgaria, Burkina, Faso, Burundi, Cambodia, Cameroon, Canada, Cape Verde, Central African Republic, Chad, Chile, China, Colombia, Comoros, Congo, Costa Rica, Cote d'Ivoire, Cyprus, Dominica, Dominican Republic, Ecuador, Egypt, El Salvador, Equatorial Guinea, Estonia, Ethiopia, Fiji, Finland, France, French Polynesia, Gabon, Gambia, Ghana, Greece, Guatemala, Guinea, Guinea-Bissau, Guyana, Haiti, Honduras, Hong Kong, Hungary, Iceland, India, Indonesia, Iran, Ireland, Israel, Italy, Jamaica, Japan, Jordan, Kazakhstan, Kenya, South Korea, Kyrgyz, Laos, Latvia, Lesotho, Luxembourg, Macao, Madagascar, Malawi, Malaysia, Mali, Mauritania, Mauritius, Mexico, Mongolia, Morocco, Mozambique, Namibia, Nepal, The Netherlands, New Caledonia, New Zealand, Nicaragua, Niger, Nigeria, Norway, Pakistan, Panama, Papua New Guinea, Paraguay, Peru, Philippines, Portugal, Romania, Rwanda, Samoa, Saudi Arabia, Senegal, Sierra Leone, Singapore, Slovak Republic, Solomon Islands, South Africa, Spain, Sri Lanka, St. Kitts and Nevis, St. Lucia, St. Vincent and the Grenadines, Swaziland, Sweden, Switzerland, Syrian, Arab Republic, Thailand, Togo, Trinidad and Tobago, Tunisia, Turkey, Uganda, Ukraine, UK, USA, Uruguay, Vanuatu, Venezuela, Zambia, and Zimbabwe.

\section{References}

1. Anderson, G.J.: Non-parametric tests of stochastic dominance in income distributions. Econometrica 64, $1183-1193(1996)$

2. Anderson, G.J.: A note on the inconsistency of tests employing point-wise comparisons for the equality of two functions. Mimeo, Economics Department University of Toronto (2001a)

3. Anderson, G.J.: The power and size of nonparametric tests for common distributional characteristics. Econom. Rev. 20, 1-30 (2001b)

4. Anderson, G.J.: Towards an empirical analysis of polarization. J. Econom. 122, 1-26 (2004a)

5. Anderson, G.J.: Making inferences about the polarization, welfare and poverty of nations: a study of 101 countries 1970-1995. J. Appl. Econ. 19, 537-550 (2004b)

6. Andrews, D.W.K.: A conditional Kolmogorov test. Econometrica 65, 1097-1128 (1997)

7. Atkinson, A.B.: The Economics of Inequality, 2nd edn. Clarendon, Oxford (1983)

8. Atkinson, A.B.: On the measurement of inequality. J. Econ. Theory 2, 244-263 (1970)

9. Atkinson, A.B.: On the measurement of poverty. Econometrica 55, 749-764 (1987)

10. Atkinson, A.B., Bourguignon, F.: The comparison of multi-dimensioned distributions of economic status. Rev. Econ. Stud. 49, 183-201 (1982)

11. Barrett, G., Donald, S.: Consistent tests for stochastic dominance. Econometrica 7, 171-103 (2003)

12. Beach, C.M., Davidson, R.: Unrestricted statistical inference with Lorenz Curves and income shares. Rev. Econ. Stud. 50, 723-735 (1983)

13. Boero, G., Smith, J., Wallis, K.F.: Sensitivity of the chi-squared goodness of fit test to the partitioning of data. Mimeo, Economics Department University of Toronto (2004)

14. Browning, M., Lusardi, A.: Household saving: micro theories and micro facts. J. Econ. Lit. 34, 17971855 (1996) 
15. Crawford, I.: Nonparametric tests of stochastic dominance in bivariate distributions, with an application to UK data. Institute of Fiscal Studies (1999)

16. Davidson, R., Duclos, J.-Y.: Statistical inference for the measurement of the incidence of taxes and transfers. Econometrica 65, 1453-1466 (1997)

17. Davidson, R., Duclos, J.-Y.: Statistical inference for stochastic dominance and for the measurement of poverty and inequality. Econometrica 68, 1435-1464 (2000)

18. Duclos, J.-Y., Sahn, D., Younger, S.: Robust multi-dimensional poverty comparisons. Mimeo, Cornell University (2001)

19. Dvoretzky, A., Kiefer, J., Wolfowitz, J.: Asymptotic minimax character of the sample distribution function and of the classical multinomial estimator. Ann. Math. Stat. 27, 352-374 (1956)

20. Esteban, J.-M., Ray, D.: On the measurement of polarization. Econometrica 62, 819-851 (1994)

21. Formby, J.P., Smith, W.J., Zheng, B., Chow, V.: Inequality orderings, normalized stochastic dominance and statistical inference. J. Bus. Econ. Stat. 18, 479-488 (2000)

22. Foster, J.E., Shorrocks, A.F.: Poverty orderings. Econometrica 56, 173-177 (1988)

23. Friedman, M.: A Theory of the Consumption Function. Princeton University Press, Princeton, NJ (1957)

24. Ibbott, P.: A test for stochastic dominance in bivariate distributions with an application to canadian living standards. Mimeo, Department of Economics, Business and Mathematics Kings College University of Western Ontario (2004)

25. Kiefer, J.: On large deviations of the empiric D.F. of vector chance variables and a law of the iterated logarithm. Pac. J. Math. 11, 649-660 (1961)

26. Kiefer, J., Wolfowitz, J.: On the deviations of the empiric distribution function of vector chance variables. Trans. Am. Math. Soc. 87, 173-186 (1958)

27. Kim, P.J., Jennrich: Tables of the exact sampling distribution of the two sample Kolmogorov-Smirnov criterion $D_{m, n}(m \leq n)$ 79-170. In: Institute of Mathematical Statistics (ed.) Selected Tables in Mathematical Statistics, vol I. American Mathematical Society, Providence, RI (1973)

28. Koshevoy, G.A., Mosler, K.: Multivariate Gini indices. J. Multivar. Anal. 60, 252-276 (1997)

29. Linton, O., Maasoumi, E., Whang, Y.-J.: Consistent tests for stochastic dominance: A subsampling approach. Mimeo, LSE (2002)

30. McFadden, D.: Testing for stochastic dominance. In: Fomby, T., Seo, T.K. (eds.) Studies in the Economics of Uncertainty: In Honor of Josef Hadar. Springer, Berlin Heidelberg New York (1989)

31. Maasoumi, E.: The measurement and decomposition of multidimensional inequality. Econometrica 54, 771-779 (1986)

32. Maasoumi, E.: A compendium of information theory in economics and econometrics. Econom. Rev. 12, 1-49 (1993)

33. Maasoumi, E.: Multidimensioned approaches to welfare analysis. In: Silber, J. (ed.) Chapter 15, Handbook of Income Inequality Measurement. Kluwer, Boston (1999)

34. Modigliani, F., Brumberg, R.: Utility analysis and the consumption function: An interpretation of cross section data. In: Kurihara, K.K. (ed.) Post Keynesian Economics. Rutgers University Press, New Brunswick, NJ (1954)

35. Phelps, E.S.: The golden rule of accumulation: a fable for growthmen. Am. Econ. Rev. 51, 638-643 (1961)

36. Rao, R.C.: Linear Statistical Inference and Its Applications. Wiley, New York (1973)

37. Sen, A.: Inequality Reexamined. Harvard University Press, Cambridge, MA (1995)

38. Tsui, K.-Y.: Multidimensional generalizations of the relative and absolute inequality indices: The Atkinson-Kolm-Sen approach. J. Econ. Theory 67, 251-265 (1995)

39. Wolak, F.A.: Testing inequality constraints in linear econometric models. J. Econ. 41, 205-235 (1989) 